\title{
Full stellar kinematical profiles of central parts of nearby galaxies ${ }^{\star}$
}

\author{
A. Vudragović, S. Samurović, and M. Jovanović
}

Astronomical Observatory Belgrade, Volgina 7, 11060 Belgrade, Serbia

e-mail: [ana;srdjan;milena]@aob.bg.ac.rs

Received 19 August 2015 / Accepted 17 June 2016

\begin{abstract}
Context. We present the largest catalog of detailed stellar kinematics of the central parts of nearby galaxies, which includes higher moments of the line-of-sight velocity distribution (LOSVD) function represented by the Gauss-Hermite series. The kinematics is measured on a sample of galaxies selected from the Arecibo Legacy Fast ALFA (Alfalfa) survey using spectroscopy from the Sloan Digital Sky Survey (SDSS DR7).

Aims. The SDSS DR7 offers measurements of the LOSVD based on the assumption of a pure Gaussian shape of the broadening function caused by the combination of rotational and random motion of the stars in galaxies. We discuss the consequences of this oversimplification since the velocity dispersion, one of the measured quantities, often serves as the proxy to important modeling parameters such as the black-hole mass and the virial mass of galaxies.

Methods. The publicly available pPXF code is used to calculate the full kinematical profile for the sample galaxies including higher moments of their LOSVD. Both observed and synthetic stellar libraries were used and the related template mismatch problem is discussed.

Results. For the whole sample of 2180 nearby galaxies reflecting morphological distribution characteristic for the local Universe, we successfully recovered stellar kinematics of their central parts, including higher order moments of the LOSVD function, for signal-to-noise above 50 .

Conclusions. We show the consequences of the oversimplification of the LOSVD function with Gaussian function on the velocity dispersion for the empirical and the synthetic stellar library. For the empirical stellar library, this approximation leads to an increase in the virial mass of $13 \%$ on average, while for the synthetic library the effect is weaker, with an increase of $9 \%$ on average. Systematic erroneous estimates of the velocity dispersion comes from the use of the synthetic stellar library instead of the empirical one and is much larger than the value imposed by the use of the Gaussian function. Only after a careful analysis of the template mismatch problem does one need to address the issue of the deviation of the LOSVD from the Gaussian function. We also show that the kurtotic parameter describing symmetrical departures from the Gaussian seems to increase along the continuous morphological sequence from late- to early-type galaxies.
\end{abstract}

Key words. catalogs - gravitation - galaxies: spiral - galaxies: elliptical and lenticular, cD - galaxies: kinematics and dynamics

\section{Introduction}

HI-blind surveys based solely on the presence of neutral hydrogen were used frequently in the past to reveal correlations between various properties of galaxies and also to find fundamental features through principal component analysis (Disney et al. 2008; Toribio et al. 2011). The HI window is unbiased with respect to galaxy luminosity, selecting all the galaxies with a measurable gas content. Such a sample is required to address important issues in the field of galaxy formation and evolution. To obtain galaxy properties through the wide wavelength range, we cross-matched our sample with the spectroscopy available from the Sloan Digital Sky Survey (SDSS) with available catalogs adding ultraviolet photometry to near-infrared (NIR) and optical photometry, extending the work of Toribio et al. (2011).

In addition to galaxy properties obtained from the photometry alone, we provide measurements of the kinematical property of galaxies, their velocity dispersion obtained from the spectra. The velocity dispersion is a commonly used proxy for many relevant parameters that cannot be observed directly, such as the

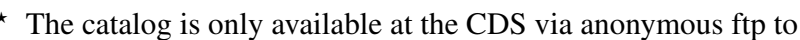
cdsarc.u-strasbg.fr (130.79.128.5) or via http://cdsarc.u-strasbg.fr/viz-bin/qcat?J/A+A/593/A40
}

virial mass and the black hole mass. It reflects a combination of rotational and random motion of stars in the galaxy. The additional parameter, namely the velocity dispersion, can be used in principal component analysis along with other properties derived from photometry as a characteristic of the central parts of the galaxies, opposite to the maximum rotation velocity available from the HI survey, which is related to the outer parts of the galaxies. They are equally important in addressing the question of galaxy formation regarding relations between different parts of the galaxy.

In this paper, we study the full line-of-sight velocity distribution (LOSVD) of the galaxies' central parts. There are currently two samples of nearby galaxies for which full LOSVD has been determined: $\operatorname{ATLAS}^{3 \mathrm{D}}$ (Cappellari et al. 2011) ${ }^{1}$ and the Hobby-Eberly Telescope Massive Galaxy Survey (HETMGS) by van den Bosch et al. (2015) ${ }^{2}$. However, the two samples are not representative of galaxy morphology: ATLAS ${ }^{3 \mathrm{D}}$ samples only early-type galaxies and HETMGS is biased towards galaxies with high central velocity dispersion, which makes them suitable for black hole mass measurements. Our sample, on the other

\footnotetext{
http: //www-astro.physics.ox.ac.uk/atlas 3d

2 http://wWw2.mpia-hd.mpg.de/ fh/hetmgs
} 
hand, is assembled from the HI-blind survey and reflects the local morphological distribution of galaxies.

The plan of the paper is as follows. In Sect. 2 we present the data sample studied, followed by the detailed description of the methodology used for the calculation of stellar kinematics in Section 3 where we especially address the errors. We pay special attention to the error estimation, since their magnitude reflects the true significance of the measured parameters; we confirm that for spectra with signal-to-noise ratio, $S / N>50$, it is possible to recover the full LOSVD (see, e.g., the studies of Emsellem et al. 2004 , for the galaxies from the SAURON ${ }^{3}$ sample $S / N>60$; Cappellari et al. 2011, for the galaxies from the ATLAS ${ }^{3 \mathrm{D}}$ sample $S / N \sim 40$; Fogarty et al. 2015, for the galaxies from the $\mathrm{SAMI}^{4}$ sample $S / N \sim 50$ ). We discuss how the Gaussian approximation of the LOSVD function induces incorrect estimates of the parameters inferred from the velocity dispersion. Even more important for the proper kinematics extraction is the usage of the proper stellar library. In Sect. 4, we carry out an extensive statistical analysis of the Gauss-Hermite $h_{4}$ parameter relevant to the question of galaxy formation and evolution, and we compare the main results of our study to the estimates found in various databases. In Sect. 5 the conclusions and a brief summary of the results are presented. In Appendix A, we present the catalog of the moments of the LOSVD measured accompanied with other relevant details, and in Appendix B we provide the queries to the databases that lead to the creation of the final sample used in this paper.

\section{Data sample}

The sample of nearby galaxies used in this work is assembled from the Arecibo Legacy Fast ALFA (Alfalfa), which is an HI-blind survey motivated by the desire to remove selection optical bias. Therefore, we started from the cross-match between Alfalfa and the SDSS DR7 spectroscopic sample (Haynes et al. 2011), requiring clean optical photometry (from SDSS DR7), including only objects classified as galaxies having reliable redshift measurements. This sample was afterwards combined with the 6th Galaxy Evolution Explorer (hereafter GALEX GR6) ${ }^{5}$ through the unique identification key searching for the closest targets. These objects are then positionally cross-matched with 2MASS All-Sky Extended Source Catalog (XSC) within the search radius of 3 arcsec. The final spectroscopic sample used in this paper has UV, optical, and NIR photometry for 2180 galaxies, with information on the gas content coming from the HI as its primary source. There is a question related to the bias concerning a possible lack of early-type galaxies in an HI survey because it is known that they contain less gas than late-type galaxies. The exact answer may be obtained only through a comparison with an unbiased survey, for example in the infrared. Unfortunately, such a survey has not yet been performed. Thus, we relied on the dependence of HI content of early-type galaxies on the environmental density by Serra et al. (2012, 2014) who searched for HI in early-type galaxies inside and outside the Virgo cluster and confirmed earlier results that HI is quite common in early-type galaxies outside the densest environments. Therefore, we do not expect any significant bias towards spiral (as gas rich) galaxies in a blind HI survey. We did a cross-match with GALEX GR6 survey and the fraction of early-type galaxies

\footnotetext{
3 http://www.strw.leidenuniv.nl/sauron

4 http://sami-survey.org

5 Data used in this work are partly based on observations made with the NASA Galaxy Evolution Explorer.
}

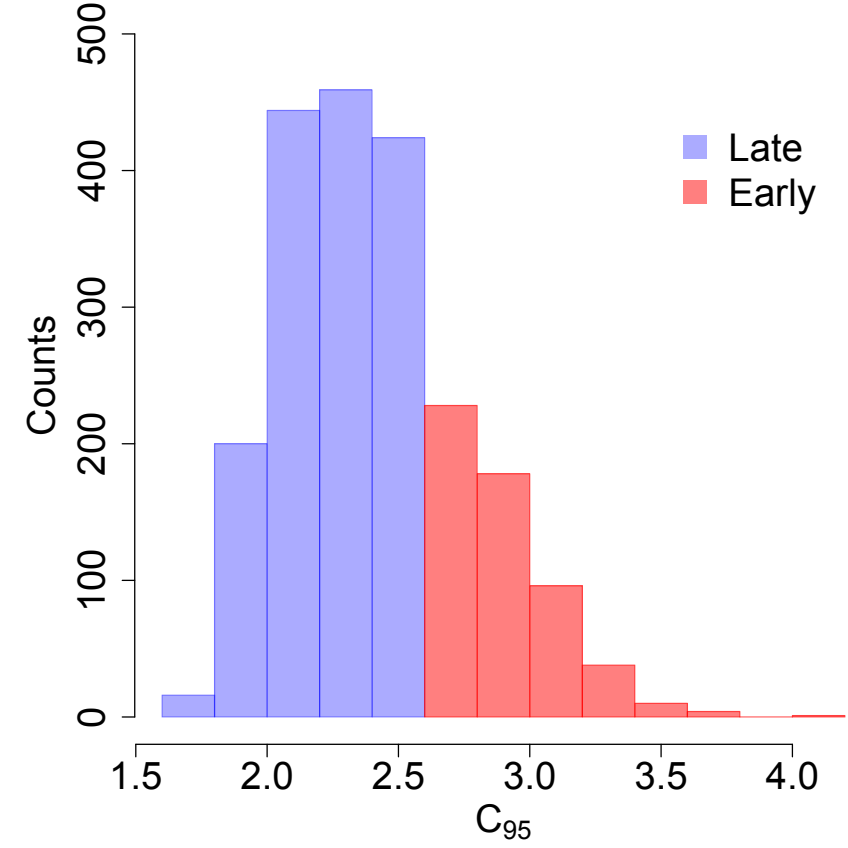

Fig. 1. Distribution of the concentration index $C_{95}$ reflecting contribution of early-type (red) and late-type (blue) galaxies using the threshold $C_{95}=2.6$. One-third of the galaxies in our sample are early-type, based on this criterion alone.

decreased by only $1 \%$, and thus this procedure did not introduce any bias with respect to late-type galaxies. All the necessary details for creating our sample are given in Appendix B.

We have inspected the morphological distribution of our galaxies using the so-called concentration index defined as the ratio of the radii containing $90 \%$ and $50 \%$ of the Petrosian flux, i.e., $C_{95}=R_{90} / R_{50}$ to separate late-type $\left(C_{95}<2.6\right)$ and earlytype $\left(C_{95} \geq 2.6\right)$ galaxies (Strateva et al. 2001). We have $26 \%$ early-type galaxies and $74 \%$ late-type galaxies divided by the cut-off value of the $C_{95}=2.6$ (Fig. 1). This proportion reflects the ratio of early- to late-type galaxies in the local Universe where approximately $1 / 3$ of all the galaxies are supposed to be early-type (Shimasaku et al. 2001) and we find it representative in this sense. However, there is a contamination from $\mathrm{Sa}$ galaxies to the pure early-type sample $(\mathrm{E}+\mathrm{S} 0)$ and a large uncertainty of \pm 1.5 class based on the use of concentration index solely (Shimasaku et al. 2001).

Huertas-Company et al. (2011) performed automatic classification of all SDSS DR7 galaxies on smaller redshifts (from the local Universe to the redshift limit of 0.25 ) with clean photometric and spectroscopic data, dividing them into early- and late-type galaxies, and then subdividing the two groups, resulting in a total of four types: elliptical (E), lenticular (S0), and spiral subtypes (Sab and Scd). According to their classification, where probabilities assigned to each type should be summed to get the probability of belonging either to early- or late-type, we have $13 \%$ early-type and $87 \%$ late-type galaxies. This should not be taken as some exact distinction between types since probabilities are continuous.

Huertas-Company et al. (2011) developed a classification method based on the learning machines (called support vector machines) that make multidimensional hyper-surfaces based on the galaxy parameters, defining regions surrounding canonical galaxy types (elliptical, lenticular, etc.). Each galaxy has its place in this volume and the distance from all the hyper-surfaces corresponding to the canonical galaxy types. The probability is 
assigned to each galaxy of being in one of the following morphological classes: E, S0, Sab, Scd. This probability is continuous and the process is completely automatic. The method was compared against several visual classifications of large galaxy samples and was proven to be robust.

We checked our morphological distribution against the Galaxy Zoo 1 data release (Lintott et al. 2008, 2011). The Galaxy Zoo project is an attempt to use as many volunteers as possible to perform classification of galaxy types "by eye" from the SDSS DR7. They were divided into spirals and ellipticals, with the subdivision of spiral galaxies that takes into account the direction of spiral arm rotation. However, we were only interested in two basic types, ellipticals and spirals, and we found that about $10 \%$ of the galaxies are early-type based on the volunteers' classifications, who reached a level of $50 \%$ (or more) votes in favor of ellipticals.

We found 782 galaxies in the HyperLeda database. Although this is less than half of our sample, we report the fraction of $13 \%$ early-type galaxies based on the numerical code for the revised (de Vaucouleurs) morphological type as defined in the RC3 cata$\log$, where early-type galaxies (both elliptical and lenticular) are designated with -5 to 0 .

We believe, following the conclusions based on the concentration index alone, that the sample used in this work reflects the local distribution of different morphological galaxy types. In other words, any other similar sample would be affected in the same manner as ours. In our final sample we have between $10 \%$ and $30 \%$ early-type galaxies.

The 7th data release of SDSS is used in this paper because it is already cross-matched with the Alfalfa survey. These spectra have a $\mathrm{S} / \mathrm{N}$ going from as low as 5 up to 80 . They cover a wavelength range of 3200-9200 $\AA$, with the spectral resolution full width half maximum $(F W H M) \sim 3 \AA$. Each of these spectra contains an error spectrum, i.e., the inverse variance suitable for creating the bad pixel mask, but also for the error propagation.

The galaxies from our sample are chosen from the redshift range $0.003<z<0.059$. This means that the upper redshift limit used in our paper is smaller than that found in the sample of Huertas-Company et al. (2011; see above).

\section{Measurements of stellar kinematics}

We have measured stellar kinematics trying to recover higher moments of the LOSVD function, taking into account the $\mathrm{S} / \mathrm{N}$ of the spectra. The deviation from the pure Gaussian function frequently used as a good approximation of the LOSVD function proves to be important, but only if the template mismatch problem is handled properly. Briefly, stellar kinematics was extracted from the galaxy spectrum by finding the best linear combination of the stellar template spectra. Apart from the template amplitudes (i.e., weights), the first four moments of the LOSVD function are calculated: radial velocity ${ }^{6}$, velocity dispersion, and the Gauss-Hermite $h_{3}$ and $h_{4}$ parameters. We use and compare the empirical and synthetic stellar library.

We anticipate here that we prefer to draw our conclusions based on the results from empirical library rather than a synthetic library because it is more reliable; they agree more closely with the results from the other empirical library used by the SDSS team counting only 32 stars. The conclusion that can be reached is that the number of template stars is less important than the

\footnotetext{
6 This is actually the offset between the stellar template and the galaxy spectrum (since the galaxy is deredshifted at the very beginning of the fitting procedure).
}

chosen stars themselves. The SDSS includes only $\mathrm{M}$ and $\mathrm{K}$ giant stars that are known to dominate the central parts of earlytype galaxies, but also the so-called "classical bulges" of spiral galaxies (i.e., bulges following the de Vaucouleurs law). On the other hand, these giant stars cannot accurately describe the central parts of spiral galaxies that are active to some extent (starforming and starburst galaxies, AGN, etc.). Therefore, additional hot stars need to be included. The Elodie library ${ }^{7}$ contains about 1000 stars of all spectral types (both hot and cold). The resulting agreement with SDSS results makes it superior to the synthetic library we use in this paper, but also to synthetic libraries used by other researchers, based, for example, on the OSSY and MaxPlank Institute for Astrophysics and Johns Hopkins University (MPA-JHU) databases (see below). We prefer SDSS measurements since they are made using observed stars and the serious problems may arise from the fact that all synthetic libraries we make comparison to yield systematically lower velocity dispersion (see below and Fig. 5). The problem does not seem to be in the number of stars they include since the SDSS library has the fewest stars of all and yet it agrees best with our empirical results. The comparison presented in Fig. 5 clearly shows that early-type galaxies (red points) are altered in the same way as other galaxy types. Also, we have carefully examined residuals for both empirical and synthetic libraries and they are quite close in the sense that they both closely resemble the galaxies they describe. The example of the fit performed using the pPXF code for the galaxy Alfalfa 240255 is shown in Fig. 2 for the case of the empirical Elodie stellar library and in Fig. 3 for the case of the synthetic stellar library.

\subsection{Gauss-Hermite coefficients}

Stellar kinematics was extracted from the SDSS DR7 spectra using the publicly available penalized Pixel-Fitting (pPXF) code (Cappellari \& Emsellem 2004). Each galaxy spectrum is represented by the linear combination of the stellar spectra broadened by the velocity dispersion reflecting the bulk stellar motion and shifted in the logarithmic space to some radial velocity, which may be recovered through the non-linear leastsquares fitting procedure. Additionally, higher moments of the LOSVD may be included in the form of the Gauss-Hermite polynomials (van der Marel \& Franx 1993) using the expression

$$
\begin{aligned}
L(w)= & \frac{1}{\sqrt{2 \pi \sigma^{2}}} \exp \left(-1 / 2 w^{2}\right) \\
& \times\left[\frac{h_{3}}{\sqrt{3}}\left(2 w^{3}-3 w\right)+\frac{h_{4}}{\sqrt{24}}\left(4 w^{4}-12+3 w^{2}\right)\right],
\end{aligned}
$$

where $w=(v-V) / \sigma(V$ being radial velocity and $\sigma$ velocity dispersion) and $h_{3}$ and $h_{4}$ are the Gauss-Hermite coefficients characterizing asymmetric and symmetric deviations from the Gaussian function, respectively. Higher Gauss-Hermite moments $h_{n}$ $(n>4)$ do exist and may be recovered, but we are only interested in the first four moments, given their relation to radial velocity and velocity dispersion through (van der Marel \& Franx 1993)

$V_{\text {corr }}=V^{\mathrm{GH}}+\sqrt{3} h_{3}^{\mathrm{GH}} \sigma^{\mathrm{GH}}$

and

$\sigma_{\text {corr }}=\sigma^{\mathrm{GH}}\left(1+\sqrt{6} h_{4}^{\mathrm{GH}}\right)$

where the superscript GH stands for Gauss-Hermite.

7 http://www.obs.u-bordeaux1.fr/m2a/soubiran/elodie_ library.html 


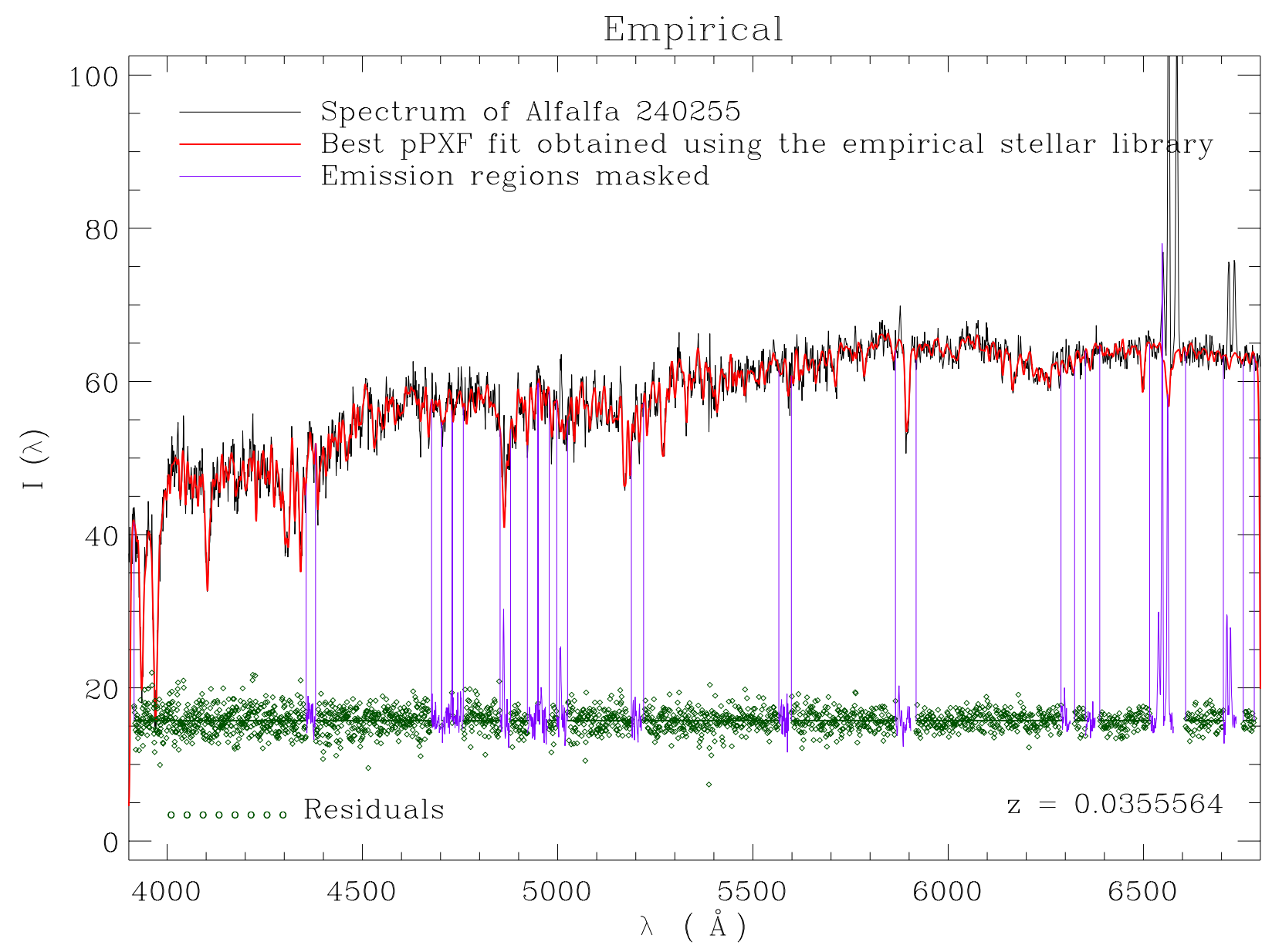

Fig. 2. SDSS spectrum of one galaxy (Alfalfa 240255) from the SDSS survey with our best pPXF fit based on the Gauss-Hermite moments overplotted in red. The masked emission regions are shown in blue and the residuals are plotted in green. The $\mathrm{S} / \mathrm{N}$ is 35 and approximately represents the mean value of the $\mathrm{S} / \mathrm{N}$ of the galaxies in our sample. The best-fitting parameters are $\sigma^{\mathrm{GH}}=120.6 \mathrm{~km} \mathrm{~s}^{-1}, h_{3}^{\mathrm{GH}}=-0.0180$, and $h_{4}^{\mathrm{GH}}=0.0400$ and the morphological type is Sab. The best pPXF fit was obtained using the Elodie empirical stellar library, and the quality of the fit is $\bar{\chi}^{2}=0.60$. The redshift of the galaxy is indicated in the lower right part of the figure, although the spectrum of the galaxy is deredshifted.

We measured stellar kinematics using both synthetic and empirical stellar library in order to address the possible template mismatch problem. We used the synthetic stellar library compiled by Tremonti et al. (2004) from the stellar population models based on the empirical library $\operatorname{STELIB}^{8}$ (Le Borgne et al. 2003). This library is embedded into the gandalf code (Sarzi et al. 2006) to work with SDSS spectra matching their resolution. There are 39 templates in total; the wavelength coverage ranges from 3200 to $9200 \AA$ and the spectral resolution is of $F W H M \sim 3 \AA$, closely following those of the SDSS spectra. These stars cover a wide range of ages and metallicities spanning from 0.005 Gyr to $10 \mathrm{Gyr}$ and three metallicities (subsolar, solar, and supersolar). We also used the empirical Elodie low resolution stellar library (Prugniel \& Soubiran 2001). It contains 1386 stars covering the wavelength range 3900-6800 and substantial space in effective temperature and metallicity $\left(3000<T_{\text {eff }}<60000 \mathrm{~K}\right.$ and $\left.-3.2<[\mathrm{Fe} / \mathrm{H}]<1.4\right)$. We note that in this paper we reduced the wavelength range for the synthetic case, i.e., for the sake of comparison of our results based on both libraries, we use the same wavelength range, from 3900 to $6800 \AA$, in both the empirical and synthetic cases. We found that all empirical spectra have gaps ${ }^{9}$, so we choose to fill these

\footnotetext{
8 http://webast.ast.obs-mip.fr/stelib

9 The gap is a region of the spectrum with missing values.
}

gaps by cubic interpolation in all the cases where the gaps were not larger than $100 \AA$ and if not more than 1 pixel is missing at the spectrum ends. This approach is justified by the fact that the pPXF code fits the whole spectrum and not particular stellar features or some part of the spectrum, so covering larger parts with "fake" values by interpolation may affect fitting results. Finally, there were 998 template stars that fulfilled the imposed criteria.

\subsection{Error estimation}

Cappellari \& Emsellem (2004) introduced the so-called bias parameter to control the true improvement of the fit since increasing the number of fitting parameters will formally reduce the $\chi^{2}$ value. The bias parameter $(\lambda)$ is closely related to the deviation of the Gauss-Hermite function from the Gaussian function $(D)$

$\chi_{\mathrm{p}}^{2}=\chi^{2}\left(1+\lambda^{2} D^{2}\right)$

where $\chi_{\mathrm{p}}^{2}$ and $\chi^{2}$ are penalized and non-biased $\chi^{2}$ values, respectively, while the deviation is expressed via the Gauss-Hermite coefficients $D^{2}=\sqrt{h_{3}^{2}+h_{4}^{2}}$ (van der Marel \& Franx 1993). This parameter can be used to penalize the solution of the LOSVD expressed in general in the form of the Gauss-Hermite 


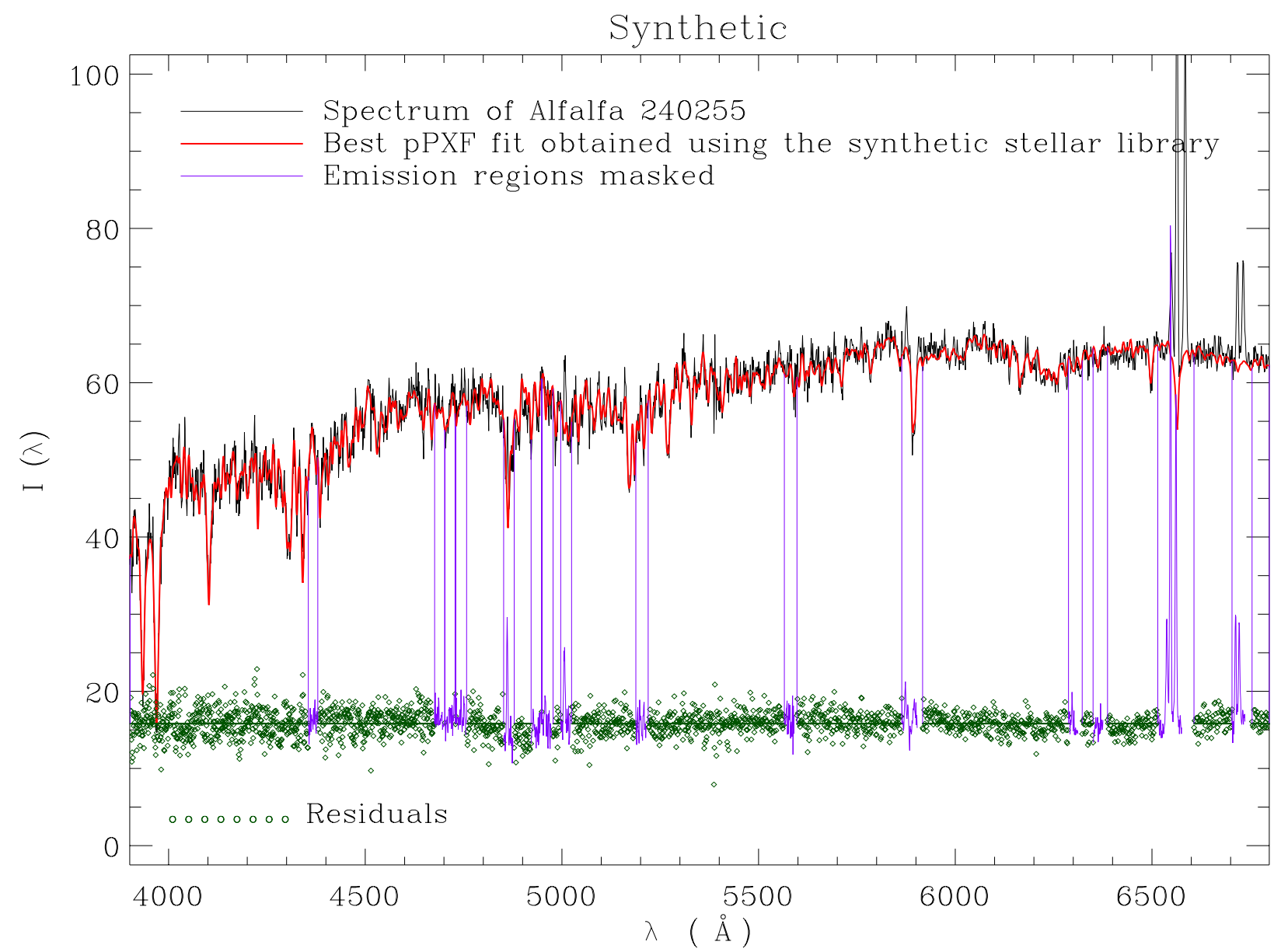

Fig. 3. SDSS spectrum of the galaxy Alfalfa 240255 shown in Fig. 2, but the best pPXF fit was obtained using the synthetic stellar library. The best-fitting parameters are $\sigma^{\mathrm{GH}}=104.1 \mathrm{~km} \mathrm{~s}^{-1}, h_{3}^{\mathrm{GH}}=-0.0228$, and $h_{4}^{\mathrm{GH}}=0.0529$ and the quality of the fit is $\bar{\chi}^{2}=0.70$. Again, the spectrum of the galaxy is deredshifted.

series toward the Gaussian function in the case of low S/N spectra. The explanation is simple: for a good fit $\chi^{2} \sim N$, so for a given $\lambda, \Delta \chi^{2}$ has to decrease by more than $N \lambda^{2} D^{2}$, implying that the parameters $h_{3}$ and $h_{4}$ improve the fit significantly. The bias needs to be determined a priori from the Monte Carlo simulations. Simply, one of the stellar template spectra from the stellar library is chosen, degraded to the S/N of the galaxy, and broadened with the input Gauss-Hermite function $\left(V_{\text {in }}, \sigma_{\text {in }}, h_{3, \text { in }}\right.$, and $\left.h_{4, \text { in }}\right)$ to resemble the galaxy spectrum of a particular $\mathrm{S} / \mathrm{N}$. The parameters of the broadening function need to be recovered by varying the bias. The adopted bias parameter is the larger value for which $h_{3}$ and $h_{4}$ moments remain well inside the scatter produced by the simulated values (see Fig. 4 for the empirical stellar template library; a very similar plot made for the synthetic stellar library is omitted for the sake of brevity). It is worth noting again that $\mathrm{S} / \mathrm{N}$ is varied from the lowest to the highest $(5<S / N<80)$ using one of the template stars. In this way, we obtain the empirical solution presented in Table 1 for the synthetic stellar library. For the empirical library, we obtain the following simple linear dependence:

bias $=0.007 S / N+0.068$.

This relation is obtained using Monte Carlo simulation on the single star adding artificial noise to mimic arbitrary S/N. For each $\mathrm{S} / \mathrm{N}$ from the galaxy sample, all the moments of the LOSVD were recovered using the bias parameter above.
Table 1. Values of the bias parameter for various $\mathrm{S} / \mathrm{N}$ for the data obtained for the case of the synthetic stellar library.

\begin{tabular}{ccccc}
\hline \hline$S / N$ & 5 & $5-25$ & $25-50$ & $50-80$ \\
\hline Bias & 0.1 & 0.2 & 0.3 & 0.4 \\
\hline
\end{tabular}

For this best bias value, from the scatter around velocity dispersion for the galaxy in question, it is possible to estimate the errors on all the measured parameters (all four moments). Figure 4 shows these Monte Carlo simulations for different $\mathrm{S} / \mathrm{N}$ and successfully recovered moments for the bias parameter found by varying its value. Each panel represents one moment of the LOSVD function given as the input value, subtracted from the simulated value for the purpose of presentation (which is why they always oscillate around zero).

We also determined the errors associated with each particular velocity dispersion for the chosen star from the Elodie library, as described above for the synthetic stellar template library. The comparison of the dispersion calculated using observed (Elodie) and synthetic stars is given in Fig. 5 .

There is an obvious systematic here: the synthetic stellar library yields lower velocity dispersion regardless of the morphological type. This disagreement is much larger than the correction of the velocity dispersion arising from the oversimplification of the LOSVD function. 

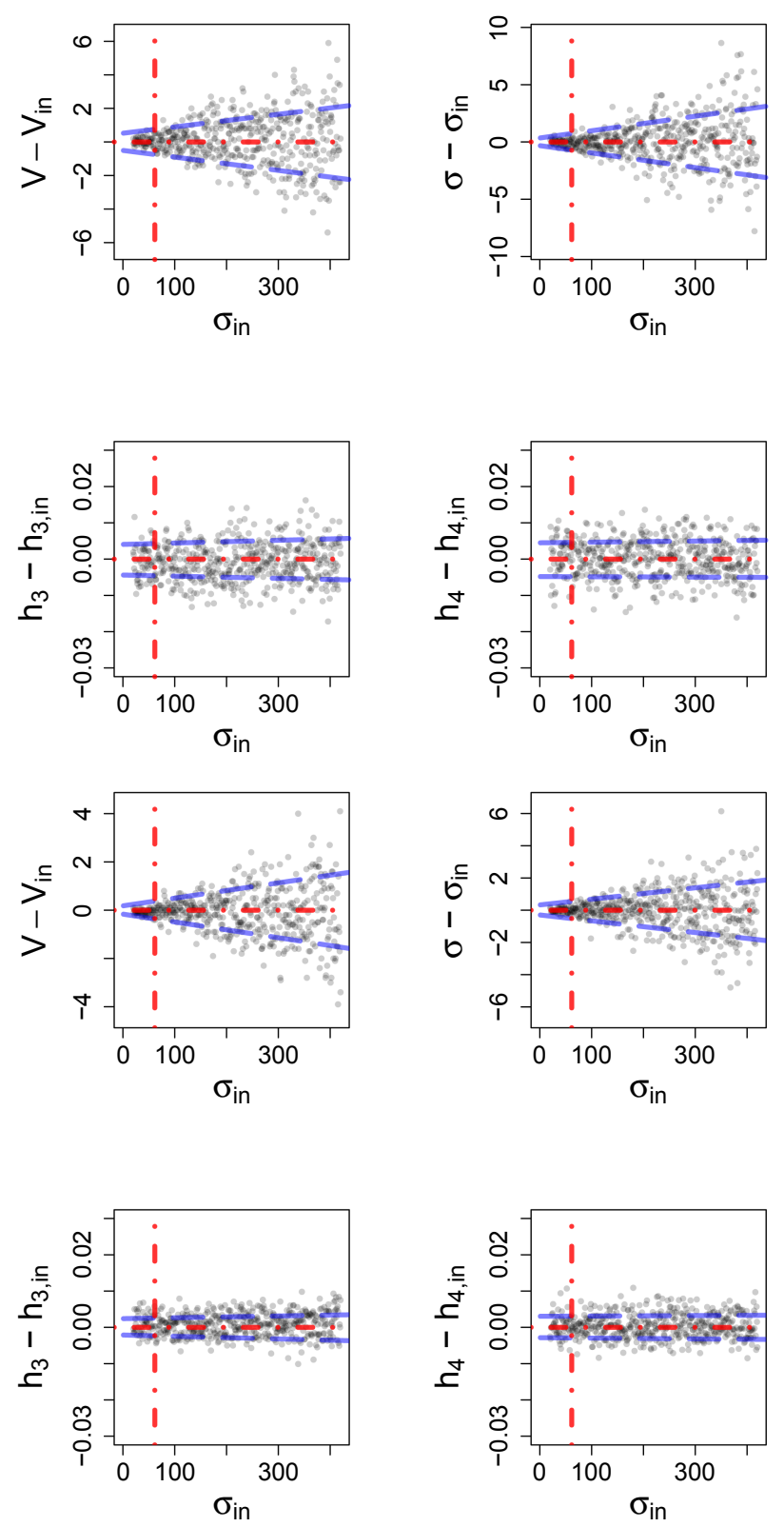

Fig. 4. Simulated values from the Monte Carlo simulations for the best bias parameter. The linear fits to the most distant points inside three times the standard deviation are plotted as blue long-dashed lines. These lines are subsequently used as errors on the associated parameters (see the text for details). Upper four plots: $S / N=30$. Lower four plots: $S / N=50$. The vertical red line is the observational resolution of $70 \mathrm{~km} \mathrm{~s}^{-1}$. The plots are for the case of the empirical Elodie stellar library.

The errors associated with the measured parameters are estimated from the Monte Carlo simulations shown in Fig. 4. We imposed the limit of 1 sigma (standard deviation) to discard the outliers, and remaining values were fitted from above (using the most distant points) with the simple linear regression. Afterwards, we made the errors symmetrical by choosing the larger error of the two. We believe that the errors calculated in this way are conservative and reflect the true significance of the parameters they are associated with.

For the synthetic stellar library, the relative errors of both Gauss-Hermite coefficients $\left(h_{3}\right.$ and $\left.h_{4}\right)$ fall below $100 \%$ for $S / N>60$, while for the empirical stellar library these errors fall

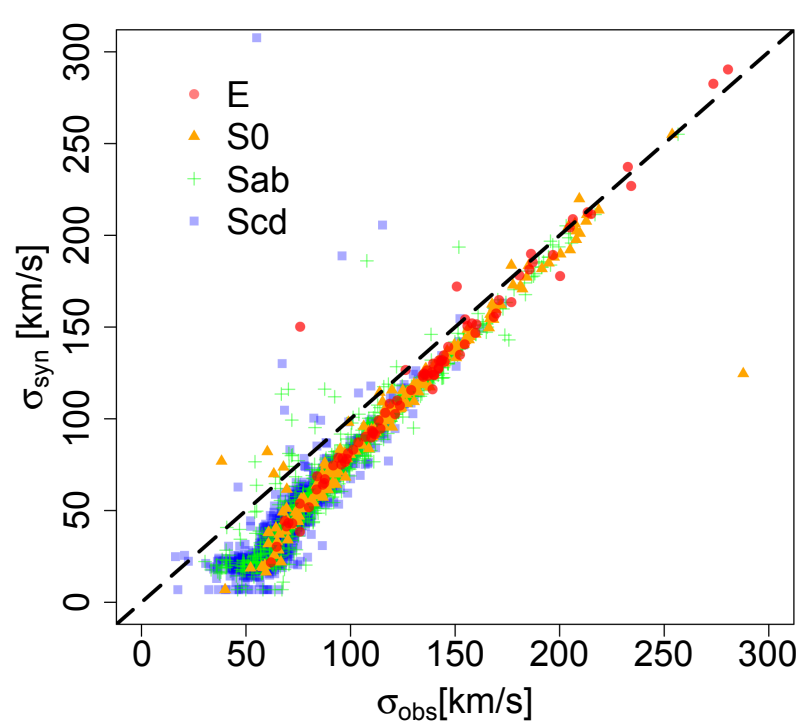

Fig. 5. Comparison between the Gaussian velocity dispersion measured using observed stars from the Elodie stellar library $\left(\sigma_{\text {obs }}\right)$ to the synthetic stellar spectra compiled using stellar population models $\left(\sigma_{\text {syn }}\right)$, with morphological types given: ellipticals $\mathrm{E}$ (red points), lenticulars S0 (orange triangles), spirals Sab (green crosses), and Scd (blue squares).

below $100 \%$ close to the median $\mathrm{S} / \mathrm{N}(S / N>45)$. The velocity dispersion relative error of the synthetic library falls below $50 \%$ for the $S / N>25$, and it falls further below $20 \%$ for $S / N>40$. In the case of the empirical library, this error is below $3 \%$ for the whole sample, and for the $S / N>35$ it is below $1 \%$.

\section{Results}

The approximation of the LOSVD function with a Gaussian function is often quite satisfactory, which is why we run the pPXF code twice, fitting the LOSVD with the Gaussian and the Gauss-Hermite functions. We were interested in inspecting the magnitude of the difference caused by this oversimplification. If significant, it may affect many empirical relations associated with the velocity dispersion. In Appendix A, we give the full description of the resulting catalog with the measured kinematics including identification keys matching various imaging surveys, along with the corrected value of the velocity dispersion. For completeness, the obtained results are given for both synthetic and empirical stellar libraries, although in the text below we rely on the dispersion coming from the empirical library $\left(\sigma_{\text {obs }}\right)$. The catalog is available in the electronic form through the CDS.

\subsection{Analysis of the Gaussian approximation}

As stated above, the pPXF code was run twice for each sample galaxy, once with the Gaussian LOSVD and again adding the higher moments of the LOSVD described by the Gauss-Hermite series. It is possible, in principle, to expect these differences to be noticeable in the SDSS galaxy spectra, since they sample the central part of the galaxy and thus show substantial velocity dispersion. Even though our sample of galaxies is dominated with spiral, star forming galaxies, their central parts are often bulges possessing this stochastic velocity component characterized by deviation from the pure Gaussian and quantified by the Gauss-Hermite coefficients. These coefficients can discriminate between different formation scenarios and are thus important for the understanding of galaxy formation and evolution. As is 


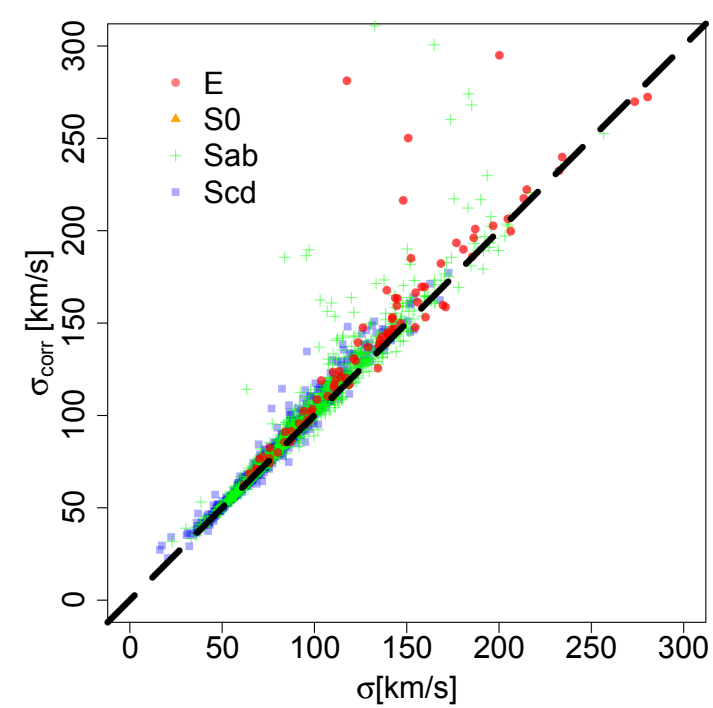

Fig. 6. Velocity dispersion corrected for the symmetric departures from Gaussian function expressed using Eq. (3), plotted to emphasize different morphological galaxy types: E (red circles), S0 (orange triangles), Sab (green crosses), and Scd (blue squares). The results are obtained using the empirical Elodie stellar library.

shown below, the $h_{4}$ parameter is useful in determining the type of anisotropy present, either radial or tangential. For example, Thomas et al. (2009) analyzed various oblate axisymmetric dynamical models for the determination of the orbital structure of intermediate mass to massive early-type galaxies in the Coma galaxy cluster and found that various orbital compositions are possible. They concluded that collisionless disk merger remnants are radially anisotropic, but they note that the lack of strong radial anisotropy would lead to the conclusion that early-type galaxies "may not have formed from mergers of discs unless the influence of dissipational processes was significant". For the study of orbits in elliptical galaxies in their outer parts we refer the reader to the numerical simulations of Dekel et al. (2005) who found that in elliptical galaxies radial orbits $\left(h_{4}>0\right)$ should dominate.

The difference plotted in Fig. 6 may not look significant, but when the ratio of two dispersions, corrected and uncorrected, is plotted (Figs. 7 and 8) using the empirical and synthetic stellar template libraries, respectively, especially taken to the power of two (the ratio of the virial masses) or to the power of four (the ratio of the black hole masses) it becomes obvious that the correction becomes significant. Figures 7 and 8 are made with a subsample of early-type galaxies $(\mathrm{E}+\mathrm{S} 0)$ to illustrate the implications of this correction for the galaxies for which virial mass calculation from velocity dispersion holds and the so-called M-sigma relation is valid.

Since these objects are true early-type galaxies, we can assume that they all have classical bulges and so the M-sigma relation holds (Kormendy \& Ho 2013):

$\log \left(M_{\mathrm{BH}} / M_{\odot}\right) \propto \beta \log (\sigma)$.

Here, the black hole mass is expressed in units of solar mass. In the seminal work of Ferrarese \& Merritt (2000), $\beta=4.8 \pm 0.5$. It is possible to see that the correction of the velocity dispersion taken to the power of four is significant $(n=4$ histogram in Figs. 7 and 8).
Empirical

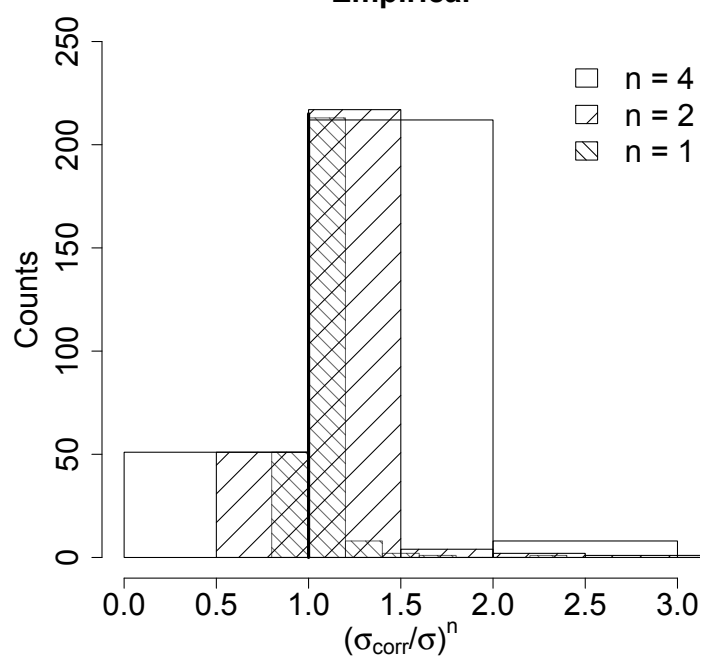

Fig. 7. Ratio of the corrected and Gaussian velocity dispersion for the sample of early-type galaxies ( $n=1$ histogram) taken to the power of 2 ( $n=2$ histogram) and $4(n=4$ histogram) to emphasize the importance of the correction when velocity dispersion is used as a proxy for either virial or the black hole mass. The empirical stellar template library was used.

\section{Synthetic}

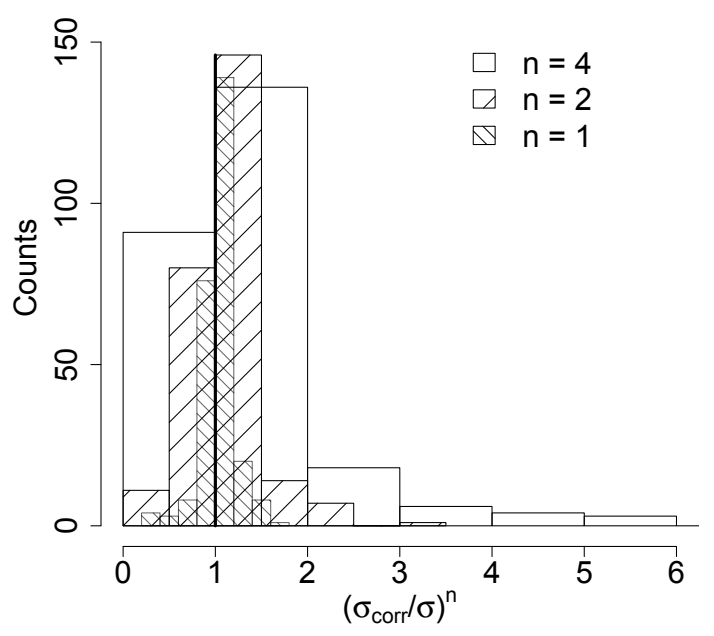

Fig. 8. As in Fig. 7, but using the synthetic stellar template library. We note that different scales were used with respect to Fig. 7.

Furthermore, following Bertin et al. (2002) the virial theorem for the stationary stellar system may be written as

$\frac{G \Upsilon L_{*}}{R_{\mathrm{e}}}=K_{V} \sigma_{0}^{2}$,

where $\Upsilon$ is the stellar light-to-mass ratio, $R_{\mathrm{e}}$ is the effective radius, and $\sigma_{0}$ is the central velocity dispersion for the aperture radius of $R_{\mathrm{e}} / 8 ; K_{V}$ is the virial coefficient given by Cappellari et al. (2006) for a sample of early-type galaxies at the redshift $z \approx 0$ to be $K_{V} \propto 5.0 \pm 0.1$. From this equation, the total dynamical mass is

$M_{\text {dyn }}=K_{V} \frac{\sigma_{\mathrm{e}}^{2} R_{\mathrm{e}}}{G}$,

where $\sigma_{\mathrm{e}}$ is velocity dispersion measured within one effective radius. Thus, the inaccurate estimate of the central velocity dispersion inevitably leads to an inaccurate estimate of the dynamical 
mass. The important point is the proportionality of this mass to the square of the central velocity dispersion (histogram labeled $n=2$ in Figs. 7 and 8 made for empirical and synthetic libraries, respectively). The mass calculated using the second or the fourth power of the velocity dispersion, approximating LOSVD with the Gaussian function in most of the cases for the objects in our sample is underestimated. The mass calculated using the second or the fourth power of the corrected velocity dispersion is larger on average than the value obtained from the dispersion inferred from the Gaussian approximation. This can be seen from the asymmetry to the right (in the direction of decreasing $\sigma_{\text {corr }} / \sigma$ ratio, above 1), implying that more galaxies tend to be more massive than the oversimplification of their LOSVD function implies (see Fig. 7). On average, the mass of early-type galaxies increases by $13 \%$ in the empirical case when the velocity dispersion is corrected according to Eq. (3). For the synthetic case, we have the same effect, only a bit smaller since the mass increases on average by $9 \%$ (see Fig. 8 ).

When the stellar library with minimal template mismatch is chosen, further corrections owing to the simplification caused by the Gaussian function have significance. However, the most important point for the reliable kinematics extraction is the minimization of the template mismatch, as can be seen in Fig. 5: we compare the dispersion measured using synthetic spectra $\left(\sigma_{\text {syn }}\right)$ with those obtained relaying on the empirical Elodie stellar library $\left(\sigma_{\mathrm{obs}}\right)$. As given above, the discrepancy is systematic, underestimating velocity dispersion by $\sim 20 \%$ above the instrumental resolution of $70 \mathrm{~km} \mathrm{~s}^{-1}$.

\subsection{Comparison to other works}

The comparison with the published velocity dispersion represented by the pure Gaussian function is shown in Figs. 9 and 10. The available measurements include the SDSS DR7 pipeline outputs and the MPA-JHU catalog. The latest database including absorption-line indices together with the velocity dispersions of the galaxies is the OSSY database ${ }^{10}$.

The overall agreement (Fig. 10) with the existing measurements is good. The observed discrepancies may exist because different stellar libraries were used by different groups, but also because of problems arising from the presence of nebular emission lines in spiral galaxies. In the low-velocity dispersion end, many SDSS DR7 measurements are missing (Figs. 9 and 10, top). The reason lies in the fact that the velocity dispersion was measured only in early-type galaxies and those galaxies whose bulges resemble early-type galaxies (the classical bulges), since stellar templates were carefully chosen to describe early morphologies (32 $\mathrm{K}$ and $\mathrm{G}$ giant stars in M67). The MPA-JHU group used the MILES empirical spectral library (Sanchez-Blazquez et al. 2006) as the basis for models generated using the population synthesis code of Bruzual \& Charlot (2003). The OSSY kinematical measurements combined specially constructed spectra using the STELIB library with Bruzual \& Charlot (2003) stellar population model spectra and in addition a set of stellar spectra based on the empirical MILES stellar library, combining both synthetic and empirical templates.

The agreement between the measurements obtained using empirical and synthetic stellar library is as expected: our empirical results closely follow empirical SDSS results, while our synthetic results agree better with the synthetic results from MPA-JHU and OSSY catalogs. We are raising concern about

\footnotetext{
${ }^{10}$ http://gem.yonsei.ac.kr/ ksoh/wordpress/
}
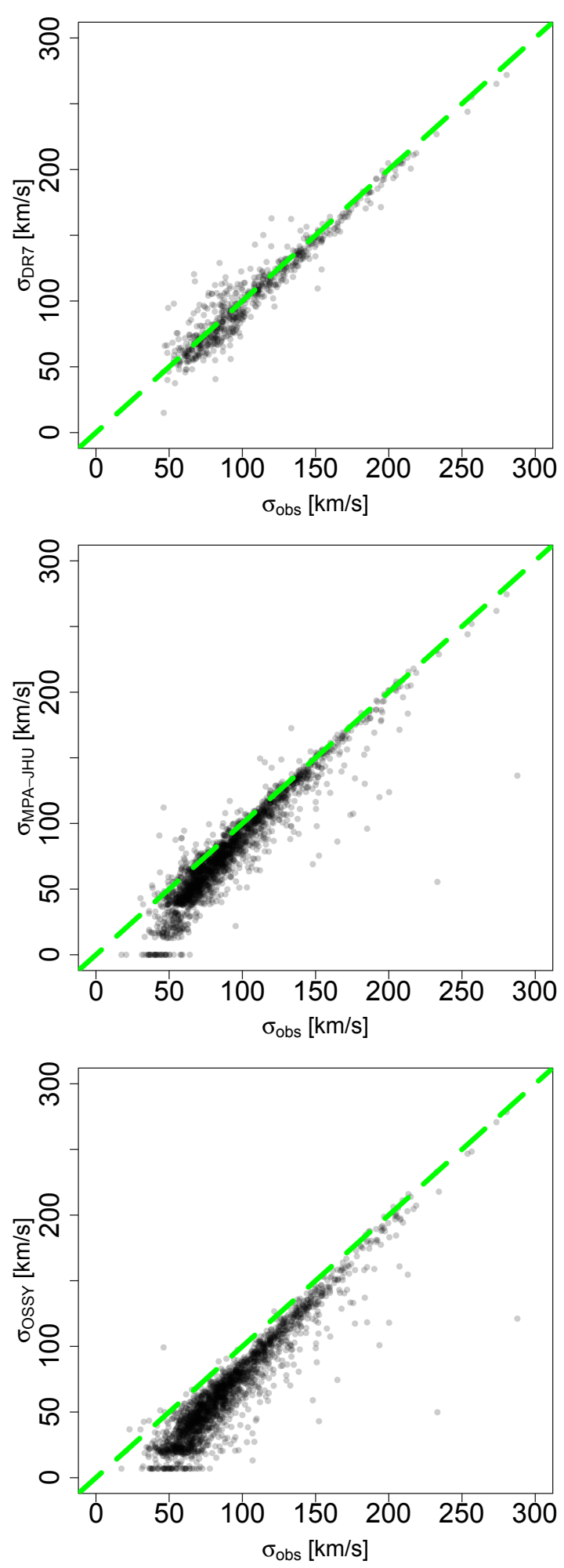

Fig. 9. Comparison of the extracted kinematics with the existing measurements of the SDSS DR7 (top), MPA-JHU (middle), and OSSY (bottom) database, respectively. The dashed line is a one-to-one correspondence. Here, the empirical templates were used.

the use of the synthetic stellar libraries, even though they are based on the observed stars. As already stated above, stellar template stars were carefully chosen by the SDSS to describe early morphologies, so all stellar libraries (both synthetic and empirical) should provide good agreement at least with these types of galaxies. However, all synthetic libraries (including the one used 

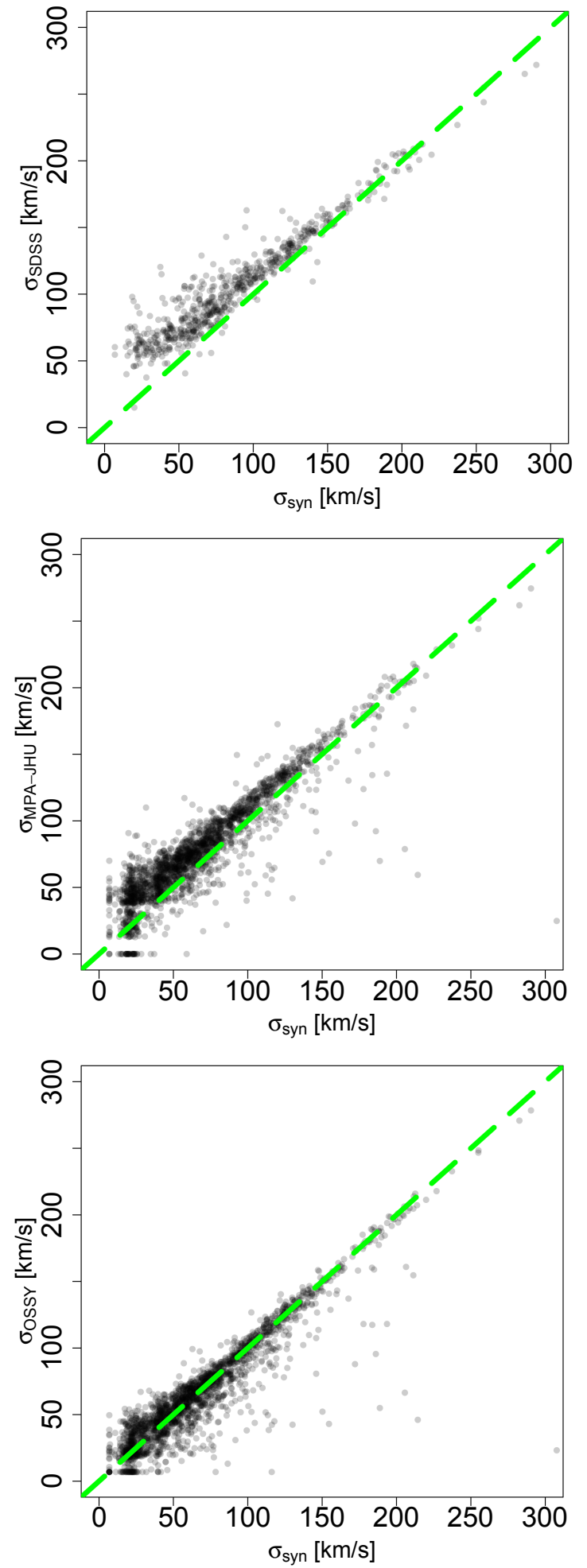

Fig. 10. As in Fig. 9 are shown, but for the synthetic stellar library.

in this paper) yield systematically lower velocity dispersions all along the morphology sequence, including early-type galaxies.

\subsection{Gauss-Hermite moments of galaxies of different morphological types}

Of particular interest to us is the Gauss-Hermite $h_{4}$ parameter which describes the stellar orbits in nearby galaxies: $h_{4}<$ 0 means that tangential orbits dominate and $h_{4}>0$ means that radial orbits dominate. In Table 2 and in Figs. 11 and 12 we

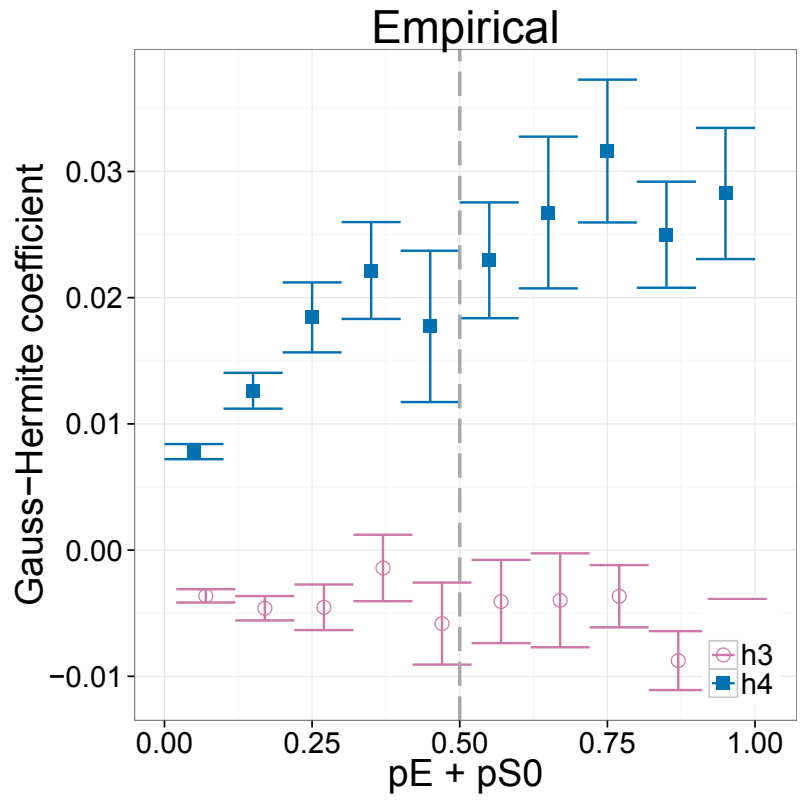

Fig. 11. Gauss-Hermite coefficients binned according to their morphological type expressed in the continuous probability of belonging to early-type galaxies $(0 \leq \mathrm{pE}+\mathrm{S} 0 \leq 1): h_{3}$ (red open circles) and $h_{4}$ (blue filled squares). The vertical dashed line $(\mathrm{pE}+\mathrm{S} 0=0.5)$ is the cut-off value dividing late-type $(\mathrm{pE}+\mathrm{S} 0<0.5)$ and early-type galaxies ( $\mathrm{pE}+\mathrm{S} 0 \geq 0.5)$. The plot is made using the empirical stellar template spectra.

present our results made with the empirical and synthetic stellar template libraries, respectively, related to the estimates of the $h_{3}$ and $h_{4}$ parameters for all the galaxies in our sample. From Fig. 11 and the upper part of Table 2, it is clear that while the $h_{3}$ parameter is approximately constant over various morphological types, the behavior of the $h_{4}$ parameter is more interesting: both for early-type $(\mathrm{pE}+\mathrm{pS} 0 \geq 0.5)$ and late-type $(\mathrm{pE}+\mathrm{pS} 0<0.5)$ galaxies there is a hint that the $h_{4}$ parameter increases as the probability $\mathrm{pE}+\mathrm{pS} 0$ increases, i.e., the value of $h_{4}$ increases going from late- to early-type galaxies. The corresponding graph for the spiral galaxies - where the probability of the galaxy being spiral on the $x$-axis is expressed through $\mathrm{pS}$ probability $(\mathrm{pS}=\mathrm{pSab}+\mathrm{pScd})-$ is omitted since the relation $\mathrm{pE}+\mathrm{pSO}=1-(\mathrm{pSab}+\mathrm{pScd})$ holds, and so it is an inverse of Fig. 11.

The consequences of using the results obtained for the empirical and synthetic cases is serious: with the empirical library we obtain a positive value of $h_{4}$ in all cases (see Table 2, top, and Fig. 11), while for the case of the synthetic library we obtain $h_{4}<0$ for some late-type galaxies and $h_{4}>0$ for early-type galaxies (see Table 2, bottom, and Fig. 12). It is interesting, however, that the trend in both cases shows an increase in $h_{4}$ from the smallest to the highest values of $\mathrm{pE}+\mathrm{pS} 0$.

We note that the plot similar to Fig. 11 made with the synthetic stellar library presented in Fig. 12 shows identical trends for both Gauss-Hermite coefficients, with the difference that the $h_{4}$ parameter remains negative for some late-type galaxies, as given above.

\subsection{Statistical tests}

Since we are more interested in the $h_{4}$ parameter, we performed full statistical analysis investigating the relation between the $h_{4}$ parameter and the morphological galaxy types. We start with the 


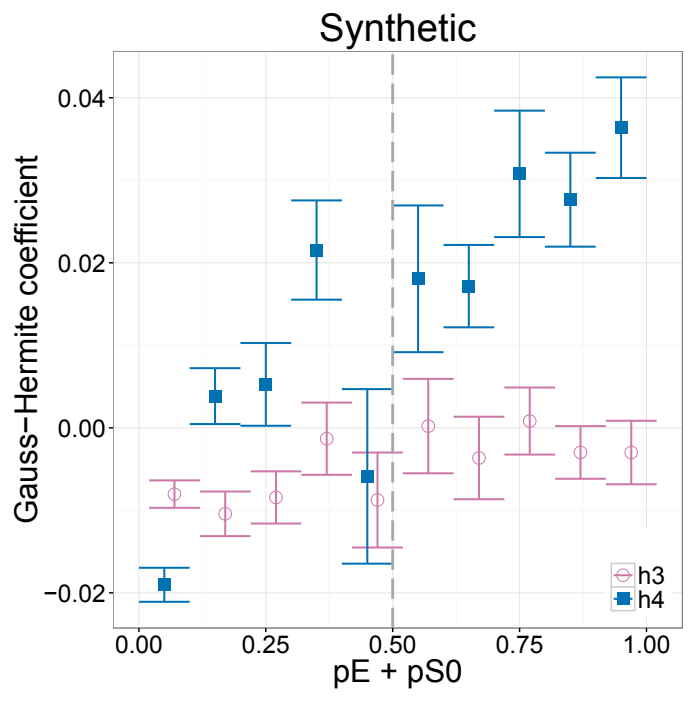

Fig. 12. As in Fig. 11, but using the synthetic stellar template library. We note the different scale on the $y$-axis with respect to Fig. 11 .

Table 2. Gauss-Hermite moments of various morphological types of galaxies (based on the empirical and synthetic stellar template spectra).

\begin{tabular}{cccccc}
\hline \hline Type & $h_{3}$ & $\Delta h_{3}$ & $h_{4}$ & $\Delta h_{4}$ & $N$ \\
\hline \multicolumn{7}{c}{ Empirical } & & & & \\
\hline 0.05 & -0.0036 & 0.0005 & 0.0078 & 0.0006 & 1254 \\
0.15 & -0.005 & 0.001 & 0.013 & 0.001 & 362 \\
0.25 & -0.004 & 0.002 & 0.018 & 0.003 & 147 \\
0.35 & -0.001 & 0.003 & 0.022 & 0.004 & 80 \\
0.45 & -0.006 & 0.003 & 0.018 & 0.006 & 50 \\
0.55 & -0.004 & 0.003 & 0.023 & 0.005 & 51 \\
0.65 & -0.004 & 0.004 & 0.027 & 0.006 & 45 \\
0.75 & -0.004 & 0.002 & 0.032 & 0.006 & 56 \\
0.85 & -0.009 & 0.002 & 0.025 & 0.004 & 57 \\
0.95 & -0.006 & 0.002 & 0.028 & 0.005 & 67 \\
\hline \multicolumn{7}{c}{ Synthetic } & & & & \\
\hline 0.05 & -0.008 & 0.002 & -0.019 & 0.002 & 1207 \\
0.15 & -0.010 & 0.003 & 0.004 & 0.003 & 330 \\
0.25 & -0.008 & 0.003 & 0.005 & 0.005 & 130 \\
0.35 & -0.001 & 0.004 & 0.022 & 0.006 & 76 \\
0.45 & -0.009 & 0.006 & -0.006 & 0.011 & 45 \\
0.55 & 0.000 & 0.006 & 0.018 & 0.009 & 45 \\
0.65 & -0.004 & 0.005 & 0.017 & 0.005 & 44 \\
0.75 & 0.001 & 0.004 & 0.031 & 0.008 & 53 \\
0.85 & -0.003 & 0.003 & 0.028 & 0.006 & 58 \\
0.95 & -0.003 & 0.004 & 0.036 & 0.006 & 67 \\
\hline
\end{tabular}

Notes. The "type" in the first column is the joint probability that a galaxy is elliptical and lenticular (pE $+\mathrm{pS} 0)$ (i.e., that the galaxy is an early-type object); the cut-off value is early-type $=\mathrm{pE}+\mathrm{pS} 0 \geq 0.5$. The total number of galaxies in the table is 2169 for the sample based on the empirical library and 2055 for the sample based on the synthetic library because the galaxies for which both $h_{3}$ and $h_{4}$ parameters could not be determined accurately were not included.

available data on four different galaxy types: E, S0, Sab, and $\mathrm{Scd}$. Then we grouped these four types into early- $(\mathrm{E}+\mathrm{S} 0)$ and late- $(\mathrm{Sab}+\mathrm{Scd})$ type classes and explored all the groups using several statistical tests.

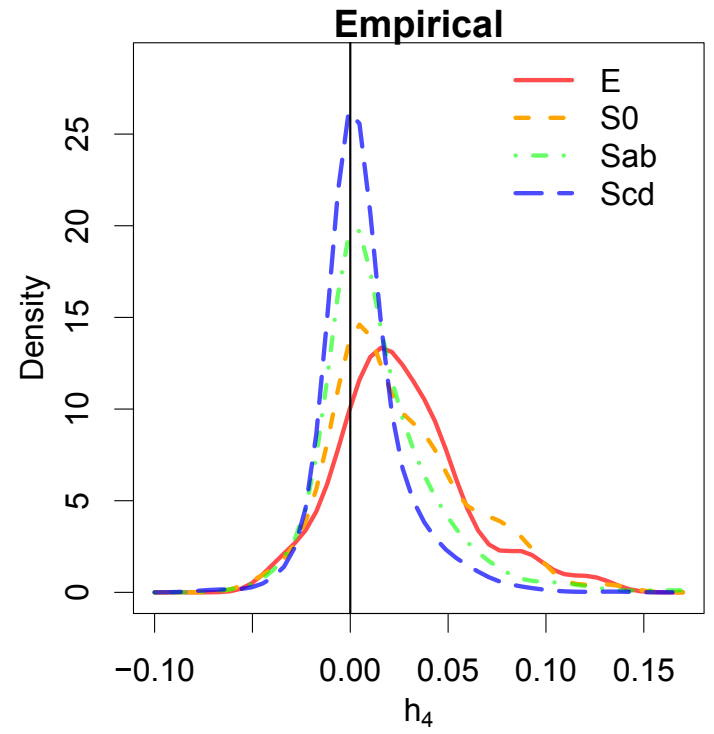

Fig. 13. Kernel density plots of the $h_{4}$ parameter of different morphological galaxy types (based on the empirical stellar template spectra): E, S0, Sab, and Scd.

We started with two main subsamples of early- and late-type galaxies. The division between types is based on the normalized probabilities with the cut-off value of 0.5 and is not exact, as given in the paper of Huertas-Company et al. (2011) who claim that "a galaxy that is $95 \% \mathrm{Sd}$ and $0.5 \% \mathrm{E}$ will still contribute to the mass function of elliptical galaxies with a weight of 0.005 ". For example, in this particular case, we considered this galaxy to be a pure spiral galaxy in this paper. The authors make a subdivision between early- and late-type galaxies using the same method, thus giving probabilities of having a E, S0, Sab, or Scd galaxy. Therefore, we performed additional statistical analyses using all of these subgroups. The kernel density plots in Figs. 13 and 14 (obtained using package $\mathrm{sm}^{11}$ available in the $\mathrm{R}$ programming language) show the following differences in the distributions of various types. In Table 4, we list the first four moments, for both libraries used, according to the standard formulas ${ }^{12}$, where the weights are inversely proportional to the errors squared, reflecting different $\mathrm{S} / \mathrm{N}$ of our spectra and the $s_{3}$ and $s_{4}$ parameters denote skewness and kurtosis, respectively. For the empirical stellar library we find that (i) all galactic types show a peak that is on the positive side, which implies the dominance of radial orbits; (ii) the $s_{4}$ of all morphological types is positive, which implies peaked distributions in all the cases; and (iii) the tails of the distributions for all morphological types described with the $s_{3}$ parameter show that the skewness of the $h_{4}$ parameter decreases, but not significantly, going from Scd to E types (visible in Fig. 13). The E and Sab galaxies in the case of the empirical libraries show the highest value of kurtosis, $s_{4}>7.00$. The plot similar to Fig. 13 made with the synthetic stellar templates (presented in Fig. 14) shows the similar behavior, but only earlytype galaxies - ellipticals ("E") and lenticulars ("SO") - show a positive mean value of the $h_{4}$ parameter $\left(s_{1}\left(h_{4}\right)>0\right)$, while for other morphological types the mean value of the $h_{4}$ parameter is negative $\left(s_{1}\left(h_{4}\right)<0\right)$ and Sab galaxies show the highest value of kurtosis $\left(s_{4}=5.164\right)$.

\footnotetext{
11 http://www.stats.gla.ac.uk/ adrian/sm

12 http://www .nag.co.uk/numeric/fl/manual/pdf/GQ1/ g01aaf.pdf
} 


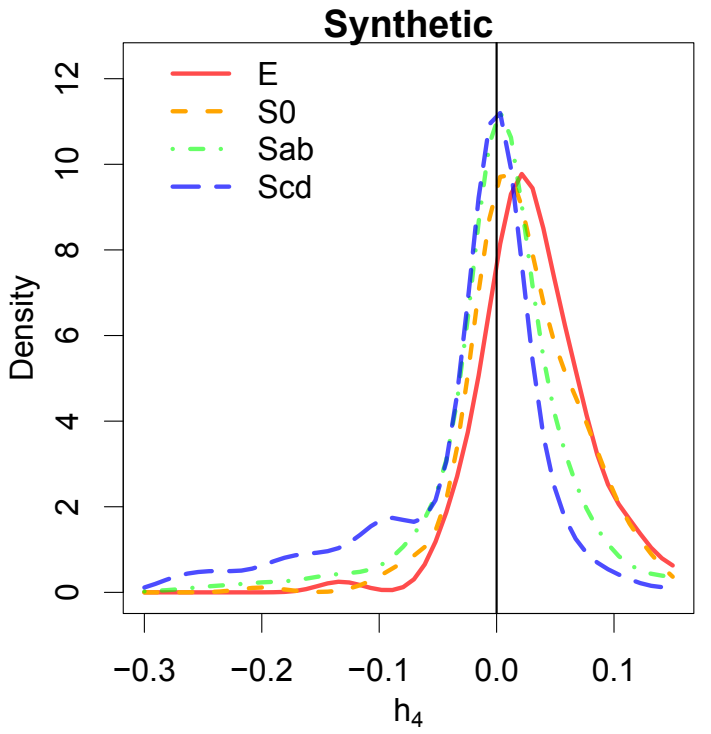

Fig. 14. As in Fig. 13, but using the synthetic stellar template library. We note that different scales were used with respect to Fig. 13.

Owing to the boundaries imposed by the pPXF code regarding the extreme values of the Gauss-Hermite coefficients, we decided to disregard galaxies with these extreme measurements from the further analysis. This slightly downsizes our sample: we are thus left with 2171 galaxies out of a total of 2180 with calculated morphology from Huertas et al. and if we exclude those galaxies having these extremes for either $h_{3}$ or $h_{4}$ Gauss-Hermite coefficients, we downsize our initial sample to 2169 galaxies (a reduction of $0.5 \%$ ) in the empirical case and to 2055 galaxies (a reduction of $5.7 \%$ ) in the synthetic case.

To inspect whether different morphological galaxy types come from the same distribution, we applied three statistical tests to the Gauss-Hermite $h_{4}$ parameter: the Anderson-Darling (hereafter AD) two-sample test, the Kolmogorov-Smirnov (hereafter KS) test, and the Cramer-von Mises (hereafter CvM) test. The AD test ${ }^{13}$ (Scholz \& Stephens 1987; Darling 1957; Anderson \& Darling 1952) is a non-parametric test that can be applied to any distribution and its main advantage over the more frequently used KS test (Hoel 1971) is its higher sensitivity to the differences presented in the tails of the distributions compared. Also, according to the test performed in Engmann \& Darling (2011) who compared the AD test with the KS test, the former also requires smaller sample sizes achieving the same prediction power as the latter, concluding that "the $\mathrm{AD}$ test is more powerful than the KS test in detecting any kind of difference between samples from two different distributions". The third frequently used test (also nonparametric and distribution-free) is the CvM test (Anderson $1962)^{14}$, but it gives less weight to the tails of the distribution (Farrell \& Rogers-Stewart 2006). Nevertheless, this test was also carried out and is in good agreement with the other two tests.

All these tests compare the so-called critical value (theoretical) calculated under the null hypothesis that two samples are taken from the same underlying distribution with the observed one, given some pre-chosen probability (the significance level at which the null hypothesis is rejected). They all compare empirical distributions, only using different criteria. A posterior

\footnotetext{
13 http://cran.r-project.org/package=kSamples

14 The CvM test was introduced as a goodness-of-fit test and was then generalized to a two-sample test.
}

Table 3. Statistical results from three different two-sided tests (AD, $\mathrm{CvM}$, and KS) performed to check whether two samples come from the same distribution for the Gauss-Hermite $h_{4}$ parameter.

\begin{tabular}{|c|c|c|c|}
\hline \multicolumn{4}{|c|}{ Empirical } \\
\hline Test & Crit. & Obs. & $p$-value \\
\hline \multicolumn{4}{|c|}{ early $(N=276)$ vs. late $(N=1893)$} \\
\hline $\mathrm{AD}$ & 1.945 & 57.5 & $8 e-25$ \\
\hline CvM & 0.034 & 0.75 & 0 \\
\hline $\mathrm{KS}$ & 0.087 & 0.29 & $2 \mathrm{e}-16$ \\
\hline \multicolumn{4}{|c|}{$\mathrm{E}(N=86)$ vs. $\mathrm{S} 0(N=190)$} \\
\hline $\mathrm{AD}$ & 1.945 & 0.280 & 0.26 \\
\hline CvM & 0.049 & 0.027 & 0.2 \\
\hline KS & 0.173 & 0.15 & 0.18 \\
\hline \multicolumn{4}{|c|}{$\operatorname{Sab}(N=913)$ vs. $\operatorname{Scd}(N=980)$} \\
\hline $\mathrm{AD}$ & 1.945 & 34.8 & $2 \mathrm{e}-15$ \\
\hline CvM & 0.030 & 0.40 & 0 \\
\hline KS & 0.062 & 0.18 & $2 \mathrm{e}-13$ \\
\hline
\end{tabular}

Synthetic

\begin{tabular}{rrrr}
\hline \multicolumn{3}{c}{ early $(N=267)$ vs. late $(N=1788)$} \\
\hline $\mathrm{AD}$ & 1.945 & 67.8 & $4 \mathrm{e}-29$ \\
$\mathrm{CvM}$ & 0.08 & 1.40 & 0 \\
$\mathrm{KS}$ & 0.088 & 0.319 & $2 \mathrm{e}-16$ \\
\hline & \multicolumn{3}{c}{$\mathrm{E}(N=84)$ vs. S0 $(N=183)$} \\
\hline $\mathrm{AD}$ & 1.945 & 1.6 & 0.07 \\
$\mathrm{CvM}$ & 0.06 & 0.05 & 0.06 \\
$\mathrm{KS}$ & 0.173 & 0.176 & 0.06546 \\
\hline & \multicolumn{3}{c}{$\mathrm{Sab}(N=859)$ vs. Scd $(N=929)$} \\
\hline $\mathrm{AD}$ & 1.945 & 47.1 & $2 \mathrm{e}-20$ \\
$\mathrm{CvM}$ & 0.08 & 1.20 & 0 \\
$\mathrm{KS}$ & 0.064 & 0.182 & $2 \mathrm{e}-13$ \\
\hline
\end{tabular}

Notes. The tests were performed for the case when the empirical template library (top) and synthetic library (bottom) were used. The listed parameters in the second and the third columns are the critical ("crit.") or tabulated values of the test and the observed ("obs.") values. The number of galaxies of this particular type used for testing is given in brackets next to the galaxy type .

probability ( $p$-value) is also calculated. The observed value ("obs." in Table 3) in the KS test is the maximum difference between the cumulative distributions of the two samples compared. The test also calculates a $p$-value from this difference accounting for the sample size in the two groups. For all the tests, the significance level is $\alpha=0.05$.

For both cases (when both empirical and synthetic libraries were used), we performed the three above-mentioned statistical tests to establish whether $h_{4}$ distributions for various Hubble types (early vs. late, E vs. S0, and Sab vs. Scd) were sampled from populations with identical distributions. If so, we can conclude that compared galaxy types are formed in a similar fashion. In both the empirical and synthetic cases, the tests agree in rejecting this null hypothesis for galaxies in two groups (early vs. late-type galaxies and Sab vs. Scd galaxies) and we conclude that they come from different distributions. For the case of ellipticals vs. lenticulars (E vs. S0) galaxies, we have a hint that they come from the same distribution because the observed values are smaller than the critical values and thus they may share a similar formation scenario. 
Table 4. The first four moments of both $h_{3}$ and $h_{4}$ distributions are given - mean, standard deviation, skewness, and kurtosis - for all galaxy types available for both libraries used.

\begin{tabular}{|c|c|c|c|c|c|}
\hline Type & $s_{1}$ & $s_{2}$ & $s_{3}$ & $s_{4}$ & $N$ \\
\hline \multicolumn{6}{|c|}{ Empirical } \\
\hline \multicolumn{6}{|c|}{$h_{3}$} \\
\hline Early & $-0.007 \pm 0.001$ & $0.0201 \pm 0.0009$ & $-0.1 \pm 0.2$ & $0.7 \pm 0.3$ & 276 \\
\hline Late & $-0.0086 \pm 0.0004$ & $0.0152 \pm 0.0002$ & $0.51 \pm 0.06$ & $5.0 \pm 0.1$ & 1893 \\
\hline $\mathrm{E}$ & $-0.008 \pm 0.002$ & $0.020 \pm 0.002$ & $0.0 \pm 0.3$ & $0.2 \pm 0.5$ & 86 \\
\hline So & $-0.006 \pm 0.002$ & $0.020 \pm 0.001$ & $-0.2 \pm 0.2$ & $1.0 \pm 0.4$ & 190 \\
\hline $\mathrm{Sab}$ & $-0.0057 \pm 0.0007$ & $0.0201 \pm 0.0005$ & $0.03 \pm 0.08$ & $1.3 \pm 0.2$ & 913 \\
\hline Scd & $-0.0093 \pm 0.0004$ & $0.0129 \pm 0.0003$ & $0.66 \pm 0.08$ & $8.0 \pm 0.2$ & 980 \\
\hline \multicolumn{6}{|c|}{$h_{4}$} \\
\hline Early & $0.025 \pm 0.002$ & $0.039 \pm 0.002$ & $1.7 \pm 0.2$ & $5.5 \pm 0.3$ & 276 \\
\hline Late & $0.0134 \pm 0.0006$ & $0.0258 \pm 0.0004$ & $2.18 \pm 0.06$ & $10.6 \pm 0.1$ & 1893 \\
\hline $\mathrm{E}$ & $0.028 \pm 0.005$ & $0.045 \pm 0.003$ & $2.4 \pm 0.3$ & $7.3 \pm 0.5$ & 86 \\
\hline So & $0.024 \pm 0.003$ & $0.034 \pm 0.002$ & $0.8 \pm 0.2$ & $0.3 \pm 0.4$ & 190 \\
\hline $\mathrm{Sab}$ & $0.016 \pm 0.001$ & $0.0319 \pm 0.0008$ & $1.96 \pm 0.08$ & $7.6 \pm 0.2$ & 913 \\
\hline Scd & $0.0109 \pm 0.0006$ & $0.0193 \pm 0.0004$ & $1.52 \pm 0.08$ & $7.0 \pm 0.2$ & 980 \\
\hline \multicolumn{6}{|c|}{ Synthetic } \\
\hline \multicolumn{6}{|c|}{$h_{3}$} \\
\hline Early & $-0.006 \pm 0.002$ & $0.026 \pm 0.001$ & $0.166 \pm 0.150$ & $1.652 \pm 0.300$ & 267 \\
\hline Late & $-0.007 \pm 0.001$ & $0.042 \pm 0.001$ & $-0.443 \pm 0.058$ & $5.716 \pm 0.116$ & 1788 \\
\hline $\mathrm{E}$ & $-0.009 \pm 0.003$ & $0.023 \pm 0.002$ & $0.271 \pm 0.267$ & $2.554 \pm 0.535$ & 84 \\
\hline SO & $-0.003 \pm 0.002$ & $0.028 \pm 0.001$ & $0.044 \pm 0.181$ & $1.239 \pm 0.362$ & 183 \\
\hline $\mathrm{Sab}$ & $-0.007 \pm 0.001$ & $0.037 \pm 0.001$ & $-0.193 \pm 0.084$ & $3.835 \pm 0.167$ & 859 \\
\hline Scd & $-0.007 \pm 0.002$ & $0.051 \pm 0.001$ & $-0.574 \pm 0.080$ & $5.206 \pm 0.161$ & 929 \\
\hline \multicolumn{6}{|c|}{$h_{4}$} \\
\hline Early & $0.024 \pm 0.003$ & $0.041 \pm 0.002$ & $-0.093 \pm 0.150$ & $4.655 \pm 0.300$ & 267 \\
\hline Late & $-0.019 \pm 0.002$ & $0.077 \pm 0.001$ & $-1.378 \pm 0.058$ & $2.488 \pm 0.116$ & 1788 \\
\hline $\mathrm{E}$ & $0.031 \pm 0.004$ & $0.036 \pm 0.003$ & $0.816 \pm 0.267$ & $4.866 \pm 0.535$ & 84 \\
\hline S0 & $0.020 \pm 0.003$ & $0.044 \pm 0.002$ & $-0.338 \pm 0.181$ & $4.130 \pm 0.362$ & 183 \\
\hline $\mathrm{Sab}$ & $-0.002 \pm 0.002$ & $0.061 \pm 0.001$ & $-1.503 \pm 0.084$ & $5.164 \pm 0.167$ & 859 \\
\hline Scd & $-0.044 \pm 0.003$ & $0.092 \pm 0.002$ & $-0.951 \pm 0.080$ & $0.483 \pm 0.161$ & 929 \\
\hline
\end{tabular}

Notes. The number of object per type is given in the last column, " $N$ ".

\section{Conclusions}

We assembled a sample of 2180 galaxies of all morphological types that reflects the morphological distribution of galaxies in the local Universe, containing about $1 / 3$ early-type and $2 / 3$ late-type galaxies, based on the concentration index alone. However, the proportion of early-type galaxies goes down to approximately $13 \%$ if a rough division is made on the basis of probabilities assigned through automatic classification of SDSS galaxies. The sample presented in this paper will be used for future studies of nearby galaxies. In this paper we restricted ourselves to the study of the kinematics of the nearby galaxies investigating their full LOSVD based on the spectra coming from the SDSS database (SDSS DR7).

Our main conclusions can be summarized as follows.

1. We confirmed that it is possible to recover the full LOSVD of nearby galaxies for which the available spectra have $S / N>50$.

2. We calculated the full LOSVD for the total of 2180 sample galaxies, which includes velocity dispersion, and the Gauss-Hermite parameters $h_{3}$ and $h_{4}$, describing asymmetric and symmetric departures from the Gaussian function, respectively. Corrections of the velocity dispersion associated with the $h_{4}$ parameter are meaningful and should be applied whenever spectra reach $S / N \sim 50$. We used two different stellar libraries to recover the LOSVD function of galaxies: a synthetic stellar library based on the empirical libraries STELIB and an empirical stellar library based on the Elodie low resolution spectra. We showed that the use of a purely Gaussian function leads in the majority of cases in our sample to erroneous estimates of important dynamical quantities, i.e., to an incorrect estimate of the black hole or virial mass inferred from the velocity dispersion for the empirical stellar library used. In this case, corrected virial mass increases by $13 \%$ on average. For the synthetic case, the mass also increases on average, but to a lower extent $(9 \%)$. However, more important than the non-Gaussian correction is the template mismatch problem. The synthetic stellar library yields systematically underestimated velocity dispersion with respect to the empirical stellar library by approximately $20 \%$.

3. We analyzed the behavior of the $h_{3}$ and $h_{4}$ parameters for the galaxies in our sample and find that while the $h_{3}$ parameter is approximately constant over all morphological types, for the $h_{4}$ parameter we have a hint of an increase from late- to early-type galaxies and this is valid for both libraries used.

4. We performed three statistical tests on the $h_{4}$ distribution comparing various morphological types and find that the distribution of early-type vs. late-type and Sab vs. Scd 
galaxies agree in rejecting the null hypothesis of the same distribution. On the other hand, for ellipticals when confronted with lenticulars (E vs. S0), we have a hint that they come from the same distribution, using both empirical and synthetic libraries, meaning that they share the similar formation scenario. This may lead to an interesting constraint important for models of galaxy evolution.

5. Using the empirical and synthetic stellar libraries leads to the conclusion that with the empirical library we obtain a positive value of $h_{4}$ in all the cases, while for the synthetic library this is not the case for all galaxy types, but holds for early-type galaxies. In both cases, there is a trend of the increase in $h_{4}$ from late- to early-type galaxies. Various statistical tests allow the common intrinsic distribution for elliptical and lenticular galaxies, using the empirical and the synthetic stellar libraries.

6. Several observational projects have successfully measured full LOSVDs of early-type galaxies out to large radii, for example, the studies of the Atlas3D project (Cappellari et al. 2011), the HETMGS project (van den Bosch et al. 2015), the CALIFA project ${ }^{15}$ (Sánchez et al. 2012), the VENGA project $^{16}$ (Blanc et al. 2013), and the MASSIVE project (Ma et al. 2014). New, recently initiated large observational projects have already made their first data releases publicly available (e.g., SAMI ${ }^{17}$; Fogarty et al. 2015) or will start producing and releasing new data very soon (e.g., MaNGA ${ }^{18}$; Bundy et al. 2015) thus providing large databases of internal kinematic structures of nearby galaxies. The studies based on these surveys will provide the full LOSVD out to more distant galactocentric radii for larger samples of galaxies and will bring the possibility for much more detailed studies of nearby galaxies of all morphological types.

Acknowledgements. We acknowledge the support from the Ministry of Educa-

tion, Science and Technological Development of the Republic of Serbia through project no. 176021 "Visible and Invisible Matter in Nearby Galaxies: Theory and Observations". We thank M. Cappellari and M. Sarzi for the help with thei software. This research made use of the NASA/IPAC Extragalactic Database (NED), which is operated by the Jet Propulsion Laboratory, California Institute of Technology, under contract with the National Aeronautics and Space Administration. This publication makes use of data products from the Two Micron All-Sky Survey, which is a joint project of the University of Massachusetts and the Infrared Processing and Analysis Center/California Institute of Technology, funded by the National Aeronautics and Space Administration and the National Science Foundation. Funding for the SDSS and SDSS-II has been provided by the Alfred P. Sloan Foundation, the Participating Institutions, the National Science Foundation, the US Department of Energy, the National Aeronautics and Space Administration, the Japanese Monbukagakusho, the Max Planck Society and the Higher Education Funding Council for England. The SDSS website is http://www.sdss.org. GALEX is operated for NASA by the California Institute of Technology under NASA contract NAS5-98034. We acknowledge the use of the HyperLeda database (http://leda.univ-lyon1.fr). The author express their gratitude to the referee for the detailed report and numerous useful suggestions and corrections that significantly improved the quality of the manuscript.

\section{References}

Anderson, T. W. 1962, Ann. Math. Statist., 33, 1148 Anderson, T. W., \& Darling, D. A. 1952, Ann. Math. Statist., 23, 193

Bertin, G., Ciotti, L., \& Del Principe, M. 2002, A\&A, 386, 149

Blanc, G. A., Weinzirl, T., Song, M., et al. 2013, AJ, 145, 138

Bruzual, G., \& Charlot, S. 2003, MNRAS, 344, 1000

Bundy, K., Bershady, M. A., Law, D. R., et al. 2015, ApJ, 798, 7

Cappellari, M., \& Emsellem, E. 2004, PASP, 116, 138

Cappellari, M., Bacon, R., Bureau, M., et al. 2006, MNRAS, 366, 1126

Cappellari, M., Emsellem, E., Krajnović, D., et al. 2011, MNRAS, 413, 813

Darling, D. A. 1957, Ann. Math. Statist., 28, 823

Dekel, A., Stoehr, F., Mamon, G. A., et al. 2005, Nature, 437, 707

Disney, M. J., Romano, J. D., Garcia-Appadoo, D. A., et al. 2008, Nature, 455, 1082

Emsellem, E., Cappellari, M., Peletier, R. F., et al. 2004, MNRAS, 352, 721 Engmann, S., \& Darling, D. A. 2011, J. Appl. Quant. Meth., 6

Farrell, P. J., \& Rogers-Stewart, K. 2006, J. Stat. Computat. Simulat., 76, 803 Ferrarese, L., \& Merritt, D. 2000, ApJ, 539, L9

Fogarty, L. M. R., Scott, N., Owers, M. S, et al., 2015, MNRAS, 454, 2050

Haynes, M. P., Giovanelli, R., Martin, A. M., et al. 2011, AJ, 142, 170

Hoel, P. G. 1971, Introduction to Mathematical Statistics, 4th edn. (New York: Wiley)

Huertas-Company, M., Aguerri, J. A. L., Bernardi, M., Mei, S., \& Sánchez Almeida, J. 2011, A\&A, 525, A157

Kormendy, J., \& Ho, L. C. 2013, ARA\&A, 51, 511

Le Borgne, J., Bruzual, G., Pello, R., et al. 2003, A\&A, 402, 433

Lintott, C. J., Schawinski, K., Slosar, A., et al. 2008, MNRAS, 389, 1179

Lintott, C., Schawinski, K., Bamford, S., et al. 2011, MNRAS, 410, 166

Ma, C.-P., Greene, J.E., McConnell, N., et al., 2014, ApJ, 795, 158

Prugniel, Ph., \& Soubiran, C. 2011, A\&A, 369, 1048

Sanchez-Blazquez, P., Peletier, R. F., Jimenez-Vicente, J., et al. 2006, MNRAS, 371,703

Sánchez, S.F., Kennicutt, R. C., Gil de Paz, A., et al. 2012, A\&A, 538, A8

Sarzi, M., Falcon-Barroso, J., Davies, R. L., et al. 2006, MNRAS, 366, 1151

Scholz, F. W., \& Stephens, M. A. 1987, J. Am. Stat. Assoc., 82, 918

Serra, P., Oosterloo, T., Morganti, R., et al. 2012, MNRAS, 422, 1835

Serra, P., Oser, L., Krajnović, D., et al. 2014, MNRAS, 444, 3388

Shimasaku, K., Fukugita, M., Doi, M., et al. 2001, AJ, 122, 1238

Strateva, I., Ivezić, Ž., Knapp, G. R., et al. 2001, AJ, 122, 1861

Thomas, J., Jesseit, R., Saglia, R. P., et al. 2009, MNRAS, 393, 641

Toribio, M. C., Solanes, J. M., Giovanelli, R., Haynes, M. P., \& Martin, A. M. 2011, ApJ, 732, 93

Tremonti, C. A., Heckman, T. M., Kauffmann, G., et al. 2004, ApJ, 613, 898 van den Bosch, R. C. E., Gebhardt, K., Gültekin, K., Yıldırım, A., \& Walsh, J. L. 2015, ApJS, 218, 10

van der Marel, R. P., \& Franx, M. 1993, ApJ, 407, 525

\footnotetext{
15 http://califa.caha.es

16 https://users.obs. carnegiescience. edu/gblancm/venga . html

17 http://sami-survey.org/data

18 https://wWW.sdss3.org/future/manga.php
} 


\section{Appendix A: The catalog}

The catalog of velocity dispersion and higher Gauss-Hermite parameters for 2180 galaxies in total with details such as morphological type (when available) is given in the electronic version of the paper and is available via Strasbourg Astronomical Data Centre (CDS).

The catalog contains the following information (column numbers are given in brackets):

(1) ALFAid - Identification from Haynes et al. (2011);

(2-3) RAdeg, DEdeg - Right ascension and declination (in degrees);

(4) GALEXid - Unique identification from GALEX GR6;

(5) pSDSSid - Unique photometric identification from SDSS DR7;

(6) SSDSSid - Unique spectroscopic identification from SDSS DR7;

(7) MASSid - Unique identification from 2MASS;

(8) $\quad z$ - Redshift from the SDSS DR7;

(9) $\mathrm{S} / \mathrm{N}-$ Signal-to-noise ratio of the spectra $^{19}$;

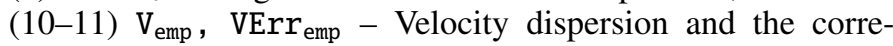
sponding error from the Gauss-Hermite LOSVD function for the empirical stellar library;

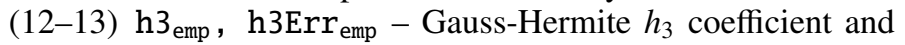
the corresponding error for the empirical stellar library;

(14-15) h4 emp, h4Err $r_{\text {emp }}$ - Gauss-Hermite $h_{4}$ coefficient and the corresponding error for the empirical stellar library;

(16-17) Vcorr $_{\text {emp }}$, VcorrErr $r_{\text {emp }}$ - Velocity dispersion corrected for the departure from the Gaussian function (see Eq. (3)) for the empirical stellar library;

(18-19) V2 ${ }_{\text {emp }}$, V2Err $\mathrm{Emp}_{\text {emp }}$ Velocity dispersion and the corresponding error for the Gaussian approximation of the LOSVD function for the empirical stellar library;

(20-21) $\mathrm{V}_{\text {synt }}$, VErr $\mathrm{V}_{\text {synt }}$ - Velocity dispersion and the corresponding error from the Gauss-Hermite LOSVD function for the synthetic stellar library;

(22-23) h3 ${ }_{\text {synt }}$, h3Err synt $_{\text {- Gauss-Hermite }} h_{3}$ coefficient and the corresponding error for the synthetic stellar library;

(24-25) h4 $4_{\text {synt }}$, h4Err synt $_{\text {- Gauss-Hermite }} h_{4}$ coefficient and the corresponding error for the synthetic stellar library;

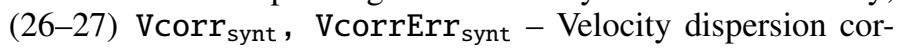
rected for the departure from the Gaussian function (see Eq. (3)) for the synthetic stellar library;

(28-29) V2 ${ }_{\text {synt }},{\mathrm{V} 2 E r r_{\text {synt }}}_{-}$Velocity dispersion and the corresponding error for the Gaussian approximation of the LOSVD function for the synthetic stellar library;

(30) $\mathrm{pE}$ - Probability of being an elliptical galaxy ${ }^{20}$;

(31) $\quad$ pSQ - Probability of being a lenticular galaxy

(32) pSab - Probability of being a spiral Sab galaxy;

(33) pScd - Probability of being a spiral Scd galaxy.

\section{Appendix B: Queries}

The final data sample was assembled going through three filters, i.e., three cross-match searches were done and we list the steps

\footnotetext{
19 The mean $\mathrm{S} / \mathrm{N}$ in three filters: $g$-, $r$-, and $i$-bands provided in the header of the spectra files.

${ }^{20}$ The probability that the galaxy belongs to one of four types (E, S0, $\mathrm{Sab}, \mathrm{Scd}$ ), used here and for the three groups below (Cols. 21-23), normalized to unity $(\mathrm{pE}+\mathrm{pS} 0+\mathrm{pSab}+\mathrm{pScd}=1)$, from the work of Huertas-Company et al. (2011). The information exists for 2171 out of the total of 2180 galaxies, i.e., $99.6 \%$ of our sample.
}

here. We started by uploading the table of Alfalfa galaxies with spectroscopic counterparts in SDSS DR7 (Haynes et al. 2011) to the SDSS Catalog Archive Server (CAS) ${ }^{21}$. First, the sample was "filtered" through SDSS DR7 tables to ensure clean photometry and reliable redshift determination:

SELECT a.AGCNr 22 as name, sp.ra, sp.dec, sp.specobjID as sdss_specobjid, sp.objID as sdss_objid, sp.z, sp.zErr

INT0 mydb.a40_SDSSDR7_noflags

FROM mydb.a40_alfalfa as a JOIN SpecPhotoAll sp ON a.SpectObjId ${ }^{23}=$ sp.specObjID

WHERE sp.zWarning $=0$ AND $\mathrm{sp} \cdot \operatorname{mode}=1$ AND sp.type $=3$ AND

$((\mathrm{sp} . \mathrm{flags} \& 0 \times 10000000) !=0)$ AND ((sp.flags \& $0 \times 8100000 \mathrm{c} 00 \mathrm{a} \theta)=0$ ) AND

$(((\mathrm{sp}$. flags \& $0 \times 400000000000)=\theta)$ or

(sp.psfmagerr_r $<=0.2)$ ) AND

$(((\mathrm{sp} . f l a g s \& 0 \times 100000000000)=0)$ or (sp.flags

$\&(0 \times 1000)=0$ )

The cross-match was carried out using unique spectroscopic identification already present in the Haynes et al. (2011) database, with some additional restrictions suggested by the SDSS DR7 team to obtain quality data. This is how we cut the initial sample of 9966 galaxies down to 6732 galaxies.

The second filter resulted in the sample of 4568 galaxies when the search for counterparts in GALEX GR6 was done, again using CAS, where the table of GALEX GR6 and SDSS DR7 cross-matches was already uploaded as xSDSSDR7. However, there are many entries for a single object, since the table was created on the basis of radial distance between objects without specifying a final counterpart. Also, we required good photometry in both the near- and far-infrared and so we used the following query to find all counterparts in the radius of 3 arcsec:

SELECT a.name, a.ra, a.dec, x.objid, a.sdss_objid, a.sdss_specobjid INT0 mydb.a40_SDSSDR7_GalexGR6

FROM photoObjAll AS $p$

JOIN xSDSSDR7 AS $\mathrm{x}$ on p.objid $=\mathrm{x}$.objid

JOIN mydb.a40_SDSSDR7_noflags AS $\mathrm{m} \mathrm{ON} \mathrm{m.objID} \mathrm{=}$ $\mathrm{x}$.SDSSObjID

JOIN photoextract AS e ON e.photoExtractID = p.photoExtractID

WHERE

distanceRank = 1 AND reverseDistanceRank = 1 AND

distance $<=3.0$ AND

e.nuvintfi != 'null' AND e.fuvintfi != 'null' AND

nuv_flux != -999 AND fuv_flux != -999

The final step was the search for 2MASS matches using IPAC Infrared Science Archive (IRSA) ${ }^{24}$. We uploaded the table with coordinates (raJ2000, decJ2000) and within a 3 arcsec radius we made a search for all the objects from $2 \mathrm{MASS}$

\footnotetext{
21 http://skyserver.sdss.org/casjobs/

22 Alfalfa galaxy ID from Haynes et al. (2011).

23 Unique spectroscopic identification in SDSS DR7 for Alfalfa galaxies in Haynes et al. (2011).

${ }^{24}$ http://irsa.ipac. caltech.edu/applications/2MASS/ PubGalPS/
} 
A. Vudragović et al.: Kinematical profiles of nearby galaxies

All-Sky Extended Source Catalog (XSC), with a suggested filter: Applying this filter results in a sample of 2180 galaxies, the final CC_flg $!=$ 'a' AND CC_flg $!=$ 'z' AND sample used in this paper.

$j_{-} f l g \_k 2 \theta f e=\theta$ AND h_flg_k20fe $=\theta$ AND

k_flg_k20fe $=0$ 